\title{
Propiedades Psicométricas del Inventario de Procesos de Autorregulación del Aprendizaje en Estudiantes Universitarios Chilenos
}

\section{Psychometric Properties of the Self-Regulated Learning Inventory in Chilean University Students}

\author{
Daniela Bruna ${ }^{1}$, María Victoria Pérez ${ }^{2}$, Claudio Bustos ${ }^{3}$ y José Carlos Núñez ${ }^{4}$
}

\begin{abstract}
Resumen
En este artículo se presenta el análisis de las propiedades psicométricas del Inventario de Procesos de Autorregulación del Aprendizaje (IPAA) en población de estudiantes universitarios chilenos. Para cumplir con este objetivo, se aplicó el instrumento a un muestra total de 780 sujetos, en su primer año de estudios, provenientes de diversos programas de pregrado. El análisis de datos se realizó mediante un análisis factorial confirmatorio y uno exploratorio. Además se analizaron los datos mediante estadísticos descriptivos, de manera de conocer la consistencia interna. Los resultados, de ambos procedimientos, evidenciaron una estructura bifactorial, con un factor general de autorregulación, que explica la mayor parte de la varianza, y 3 subfactores (Planificación, Ejecución y Evaluación), coherentes con la teoría. Además, todos los factores presentaron adecuados índices de confiabilidad. Se concluye que el IPAA es un cuestionario válido y confiable para ser aplicado a esta población.
\end{abstract}

Palabras clave: Teoría Sociocognitiva del Aprendizaje, autorregulación del aprendizaje, planificación, ejecución y evaluación del estudio, estudiantes universitarios

\begin{abstract}
This article presents the psychometric properties analysis of the Self- Regulated Learning Inventory, in Chilean university students. To achieve this aim, the instrument was applied to 780 subjects, in their first year of studies, which belong to different undergraduate programs. Data analysis was carried out using exploratory and confirmatory factor analysis. The results of both procedures showed a bifactor structure, with one general factor of self- regulation, and 3 subfactors (Forethought, Perfomance, Self- reflection), consistent with the theory. In addition, all the factors evidence adequate reliability indexes. It is concluded that IPAA is valid and reliable questionnaire, which can be applied to this population.
\end{abstract}

Keywords: Sociocognitive Theory, self- regulated learning, planning, forethought and self- reflection of study, higher education students

\footnotetext{
${ }^{1}$ Doctor en Psicología. Centro de Investigación y Mejoramiento de la Educación (CIME), Facultad de Psicología. Universidad del Desarrollo. Doctorado en Psicología. Departamento de Psicología. Universidad de Concepción. Ainavillo 456. Concepción, Chile. Tel.: +56 412268334 Correo: dbrunaj@udd.cl

${ }^{2}$ Doctor en Psicología. Departamento de Psicología. Universidad de Concepción. Tel.: +56 41 2203957. Correo: marperez@udec.cl

${ }^{3} \mathrm{Mg}$. En Psicología Educacional y Mg. en Estadística Aplicada. Doctorado en Psicología. Departamento de Psicología. Universidad de Concepción. Tel.: +56 412268334. Correo: clbustos@udec.cl

${ }^{4}$ Doctor en Psicología. Departamento de Psicología de la Universidad de Oviedo, España. Tel.: +34 985103224. Correo: jcarlosn@uniovi.es
} 


\section{Introducción}

La autorregulación del aprendizaje ha adquirido gran relevancia en el estudio del aprendizaje, especialmente en la Educación Superior (Boekaerts \& Cascallar, 2006; Cassidy, 2011; Elvira-Valdés, \& Pujol, 2012; Lanz, 2006). Una de las explicaciones de este especial interés, es que se trata de una habilidad asociada a la tradición de "Lifelong Learning", o "Aprendizaje para toda la vida", la cual ha impregnado fuertemente la investigación y políticas publicas educacionales a nivel internacional. Éste se entiende como un proceso continuo que estimula y empodera a los individuos a seguir aprendiendo conocimientos, valores y habilidades a lo largo de todas las etapas del ciclo vital (Watson, 2003). El énfasis está puesto en aprender a aprender, basándose en que los estudiantes aprendan a conocer, a hacer, a vivir con otros y a ser (Delors, 1996; European Comission, 2001). En este contexto, la autorregulación del aprendizaje es una herramienta que permitiría a los sujetos continuar aprendiendo, gracias al desarrollo de una capacidad para planificar procesos, monitorear el desempeño mientras éstos se están llevando a cabo, y autoevaluar los resultados, para llevar a cabo mejoras en futuras situaciones.

La autorregulación, desde la Teoría Sociocognitiva del aprendizaje (Panadero \& Alonso-Tapia, 2014), se trata de un proceso proactivo, de carácter iterativo y auto dirigido, en el que los aprendices transforman sus habilidades mentales en habilidades académicas (Zimmerman, 2002). Los estudiantes conducen sus pensamientos, sentimientos y conductas hacia el logro de metas de aprendizaje impuestas de manera personal, desplegando estrategias relacionadas con las tareas que deben llevar a cabo (Pintrich, 2000). Pueden hacerlo debido a que se encuentran conscientes de sus fortalezas y sus debilidades. De esta manera, logran monitorear, regular, controlar y evaluar el cumplimiento de los objetivos, y así mejoran su efectividad (Boekaerts \& Cascallar, 2006). La autorregulación incluye habilidades de metacognición, ya que permite al aprendiz pensar sobre sus propios procesos cognitivos, además de creencias de autoeficacia y de agencia personal, así como procesos motivacionales y conductuales (Zimmermann, 1995).

Se trata de un proceso importante de investigar y fomentar en los estudiantes, ya que al desarrollarse, otorga a los adultos la oportunidad de continuar aprendiendo habilidades y herramientas luego de terminada la educación escolar y universitaria, por ejemplo, cuando asumen nuevas funciones en el trabajo (Zimmerman, 2002).

La autorregulación del aprendizaje media la relación entre el contexto, las características del aprendiz y el desempeño (Pintrich, 2004), y explica de mejor manera, que la capacidades cognitivas, el logro académico de un estudiante (Zimmerman, 2002). Por esto, es vista como un mecanismo para explicar las diferencias en el logro entre los estudiantes y como medio para mejorar estos alcances académicos (Schunk, 2005). Su fomento podría ayudar a los problemas de deserción universitaria, debido a que se está asociada a ajuste académico en estudiantes universitarios de primer año. Esto implica que los estudiantes con mayores niveles de autorregulación se adaptan de manera más fácil a los desafíos que les impone la universidad (Cazan, 2012).

Estos antecedentes ponen en relieve el hecho de que los estudiantes deberían practicar y desarrollar la autorregulación durante todas las etapas educacionales (Kistner, Rakoczy, Otto, Dignath- van Ewijk, Büttner, \& Klieme, 2010), ya que se ha concluido que es posible de entrenar. (Boekaerts \& Cascallar, 2006; Schunk, 2005). Ejemplo de esto es el estudio de meta- análisis realizado por Dignath, Buettner \& Langfeldt (2008), en que a partir de la revisión de 48 interveciones realizadas en nivel primario, concluyeron que éstas son eficaces. A nivel de Educación superior, destacan los trabajos de Cerezo, Núñez, Rosário, Valle, Rodríguez, \& Bernardo (2010), quienes realizaron una revisión descriptiva de los programas existentes para promover el aprendizaje autorregulado en educación superior, con la particularidad de que sólo organizaron aquellos programas en los que se utilizó una modalidad de e- learning, además del estudio Núñez et al (2011), quienes diseñaron e implementaron un programa de entrenamiento en estrategias de aprendizaje autorregulado. El 
diseño utilizado fue cuasi- experimental con medidas de pre y post test. Los resultados fueron positivos, concluyendo que el programa fomentaba la capacidad de autorregulación de los estudiantes. Sumado a estos programas, igualmente se ha trabajado con estudiantes universitarios provenientes de áreas específicas del conocimiento, por ejemplo con estudiantes de la carrera de Psicología, de primer año (Cazán, 2013) y de Pedagogía (Inan \& Yüskel, 2010; Perry, Hutchinson, \& Thauberger, 2008).

Esta evidencia derivó hacia el desarrollo de intervenciones para fomentar esta habilidad en diferentes niveles educativos (Dignath van Ewijk \& Büttner, 2008; Núñez, Rosário, Vallejo, \& González-Pienda, 2013), siendo el interés aún mayor en educación superior, ya que si bien se supone que los estudiantes deberían llegar con la capacidad autorregulatoria a la universidad, la evidencia muestra que esto no ocurre así (Cazan, 2013; Cerezo, Núñez, Rosário, Rodríguez, \& Bernardo, 2010; Cerezo, Bernardo, Esteban, Sánchez, \& Tuero, 2015; Inan \& Yüskel, 2010; Kistner, Rakoczy, Otto, Dignath-van Ewijk, Büttner, \& Klieme, 2010; Perry, Hutchinson \& Thauberger, 2008; Rosário, et al., 2010). De esta forma aparece la necesidad de contar con instrumentos con adecuadas propiedades psicométricas de validez y fiabilidad, que permitan medir esta variable y generar diferentes líneas de investigación, especialmente en educación superior. Entre éstos, la descripción del nivel de autorregulación del aprendizaje en los estudiantes universitarios, la relación entre autorregulación del aprendizaje y variables críticas del proceso de aprendizaje, y la valoración del impacto de intervenciones de fomento de la autorregulación del aprendizaje en los estudiantes.

\section{Medición de la Autorregulación del Aprendizaje a través del Modelo de Barry Zimmerman: Inventario de Procesos de Autorregulación del Aprendizaje (IPAA).}

El modelo teórico más aceptado para explicar el aprendizaje autorregulado, dentro de la tradición sociocognitiva, es el de Barry Zimmerman (2002). Este autor lo define como un proceso con 3 fases cíclicas: Preparación, Desempeño y Autorreflexión. A continuación se explican estas fases siguiendo la propuesta de Zimmerman (2002).

La primera es la Fase de Preparación o disposición, la cual ocurre previo a la actividad de aprendizaje. En ésta se despliegan dos procesos: Análisis de la Tarea y Automotivación. En el Análisis de la Tarea se ejecutan dos subprocesos. En primer lugar se establecen las metas y en segundo lugar se realiza una planificación estratégica. El segundo proceso, de Automotivación, deriva de las creencias que los estudiantes poseen acerca del aprendizaje, e involucra cuatro subprocesos cognitivos: creencias de autoeficacia, expectativas acerca de los resultado, interés intrínseco y orientación al aprendizaje como meta.

La segunda es la Fase de Desempeño, que se despliega mientras se lleva a cabo la tarea conductualmente. Ésta implica dos procesos: Autocontrol y Autoobservación. El Autocontrol se refiere al despliegue de métodos y estrategias específicas que fueron seleccionados en la etapa anterior de preparación. La Autoobservación se refiere al hecho de tomar conciencia del proceso de estudio mientras se está llevando a cabo.

La tercera es la Fase de Autoreflexión, en la que incluye dos procesos: Autojuzgamiento y Autoreacción. En el autojuzgamiento ocurren dos subprocesos: la autoevaluación y la atribución causal. En el autojuzgamiento el aprendiz compara su desempeño con ciertos estándares, por ejemplo el desempeño anterior en situaciones similares, o el de sus compañeros. La atribución causal, por su parte, se trata de las creencias acerca de las causas de los errores y éxitos. Por su parte, La autorreacción incluye dos subprocesos: la autosatisfacción y afectos positivos, y las reacciones defensivas $\mathrm{O}$ adaptativas. $\mathrm{La}$ autosatisfacción permite aumentar la motivación, incrementando los futuros esfuerzos por aprender. En relación a las reacciones, se ha encontrado que las respuestas adaptativas son aquellas que permiten ajustar los comportamientos de manera de aumentar la efectividad del método de aprendizaje, mientras que en las respuestas desadaptativas, el estudiante se esfuerza por proteger la autoimagen, evitando las oportunidades para aprender y mostrar el desempeño personal. A continuación se muestra una figura del modelo de autorregulación traducida del original del autor (Zimmerman, 2002). 


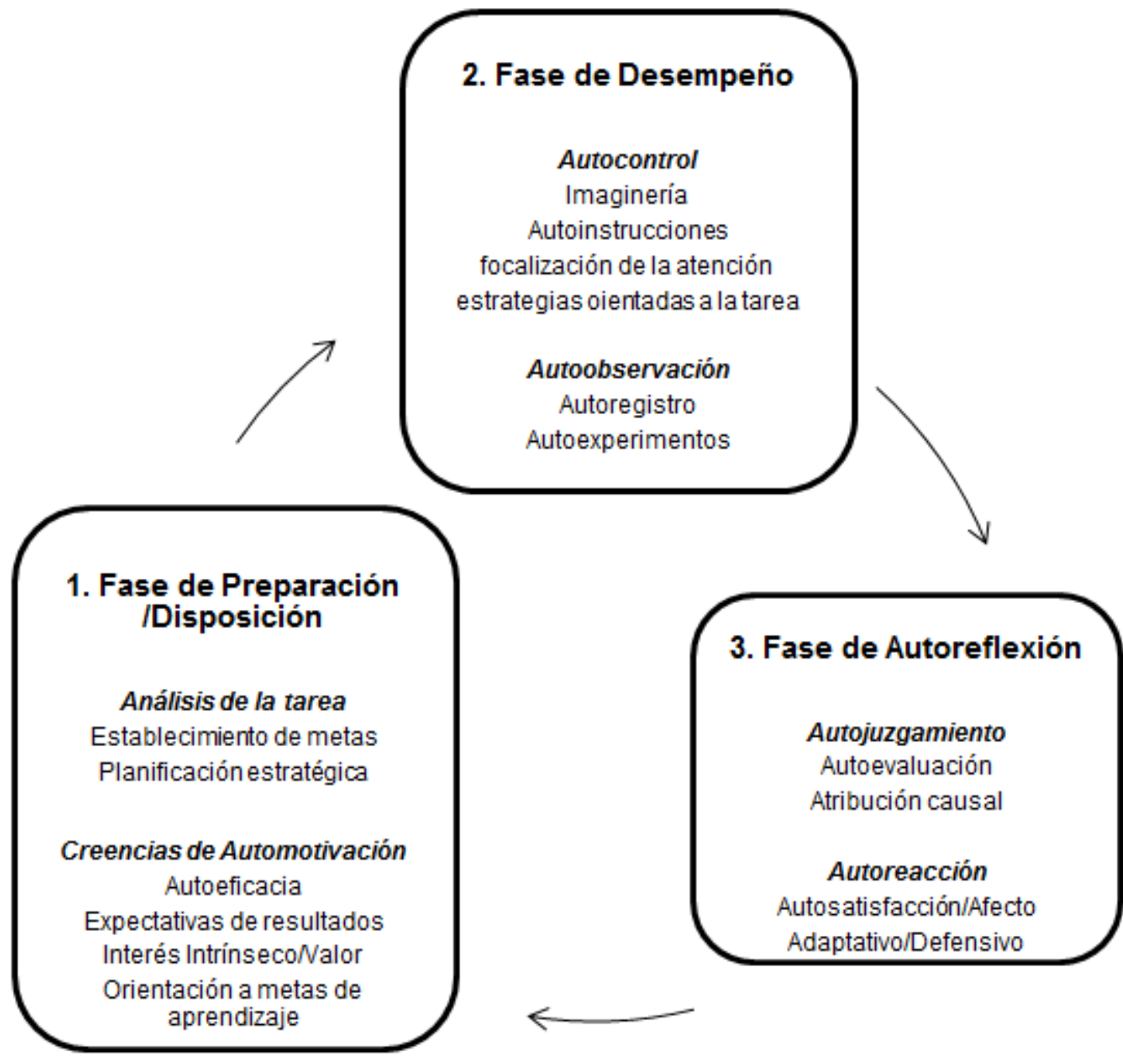

Figura 1. Modelo de Autorregulación del Aprendizaje de Barry Zimmerman (2002)

Basándose en este modelo, Rosário et al (2007), equipo conformado por investigadores portugueses y españoles, diseñaron un instrumento para medir autorregulación del aprendizaje en estudiantes universitarios, llamado IPAA. Esta escala ha sido utilizada para evaluar el impacto de múltiples intervenciones, tanto a nivel nacional, como internacional, no encontrándose la misma estructura factorial que la original (Cerezo, Núñez, Rosário, Valle, Rodríguez, \& Bernardo, 2010; Núñez et al, 2011; Pérez, Díaz, GonzálezPienda, \& Núñez, 2011, Rosário et al., 2010; 2012).

Este instrumento se encuentra en la tradición de medición de la autorregulación como aptitud (Winne \& Perry, 2000). Se trata de un cuestionario de autorreporte (Torrano \& González, 2004), lo que es un ventaja debido a que es práctico en el diseño, de fácil aplicación y permite el uso de metodologías cuantitativas complejas para el análisis de los datos (Núñez, Amieiro, Álvarez, García, \& Dobarro, 2015). Esto lo hace apropiado para investigaciones que intentan estudiar grandes muestras, evidenciar niveles de cambio en esta habilidad y evaluar el impacto de programas de mejora.

Si bien existen otros, como "The Learning and Study Strategy Inventory" (LASSI) (Catañeiras, Guzmán, Posada, Ricchini y Strucchi, 1999; Weinstein, 1987) y "The Motivated Strategies for Learning Questionnaire" (Pintrich, Smith, García, \& Mc Keanie, 1991), éstos han sido creados en culturas muy diferentes, y tienen la dificultad de su extensión (77 y 81 ítems, respectivamente), lo que complejiza el proceso de aplicación a la población de estudiante universitarios.

El presente estudio tiene por objetivo encontrar evidencias de validez en este instrumento de evaluación del proceso de autorregulación del aprendizaje en estudiantes universitarios. El IPAA (Rosário et al., 2007) ha 
mostrado adecuadas propiedades psicométricas, con población de estudiantes universitarios españoles. Además es de fácil y rápida administración. Su adaptación puede aportar en la línea de evaluación de programas, midiendo sus efectos, permitiendo no sólo realizar descripciones y diagnósticos de esta variable, si no la valoración de los resultados obtenidos en intervenciones que buscan su promoción (Martorell \& Gómez, 2010). Junto con esto, ayudaría a llenar un vacío asociado a la falta de escalas que midan este constructo en la realidad nacional $y$ Latinoamericana.

\section{Método}

\section{Participantes}

Se encuestó a un total de 780 estudiantes seleccionados a través de un muestreo de tipo no probabilístico por accesibilidad, provenientes de 16 programas de pregrado de 2 universidades de la Región del Bío Bío en Chile, una tradicional y una privada. De estos 480 fueron mujeres $(61.5 \%$ de la muestra) y 300 hombres $(38.5 \%$ de la muestra). La selección de las universidades intentó tomar en cuenta los dos tipos de instituciones existentes en el país: tradicionales y privadas, de manera de lograr un muestreo representativo de la realidad. Las universidades tradicionales, son aquellas que reciben fondos públicos, estatales, directamente, mientras que las privadas, han sido creadas por el sector privado (OCDE, 2009). Ambas instituciones seleccionadas se rigen por el Sistema de Admisión del Consejo de Rectores de Chile. Esto implica que los estudiantes rinden la Prueba de Selección Universitaria (PSU), para poder ingresar, presentando perfiles estudiantiles y puntajes promedio en esta prueba similares.

En relación con los programas, todos son de pregrado con una duración de 5 años y otorgan grado académico de licenciatura y título profesional, (OCDE, 2009). Se intentó representar a todas las áreas del conocimiento en ambas instituciones de Educación Superior. La Tabla 1 resume los programas participantes del estudio, para ambas instituciones y la distribución de sujetos por género. Los cuestionarios fueron aplicados en asignaturas del segundo semestre del primer año de los programas de estudio. 694 (89\%) estudiantes se encontraban cursando su primer año de estudios, 72 (9.2\%) el segundo, 9 $(1.1 \%)$ el tercero, $2(0.3 \%)$ el cuarto, mientras que de $3(0.4 \%)$ no se cuenta con información. La media de edad de los sujetos fue de 19 años y 6 meses, con un mínimo de 18 y un máximo de 32.

\section{Instrumento}

El IPAA (Rosário et al., 2007) está conformado por 12 ítems organizados en 3 dimensiones, representativas de las 3 fases del proceso de autorregulación del aprendizaje de Zimmerman (2002): a) Planificación, b) Ejecución, y c) Evaluación. Los ítems se puntúan en una escala Likert de 1 a 5. El Alpha de Cronbach para la escala total es de 0.87. La Tabla 2 presenta el instrumento con sus dimensiones e ítems.

\section{Procedimiento}

En primer lugar se confeccionó el consentimiento informado que debían leer y firmar los participantes. Éste daba cuenta de los objetivos de la investigación, la confidencialidad de la información entregada y el carácter voluntario de la participación en ésta.

Luego se preparó el formato de aplicación del inventario y se procedió a aplicar una entrevista cognitiva (Smith Castro \& Molina, 2011) a 3 estudiantes universitarios de primer año con rendimiento académico heterogéneo entre sí (uno sobre la media, uno en la media y uno bajo la media). El objetivo de la aplicación de esta herramienta fue evaluar la compresión que tenían los sujetos de los ítems del instrumento. Los resultados mostraron que los estudiantes entendían adecuadamente los reactivos y su sentido. Después, con el fin de determinar el tiempo requerido para la aplicación, se realizó una aplicación piloto a otros 3 estudiantes.

Posteriormente, se llevó a cabo la aplicación definitiva a los participantes, la cual duró 3 semanas y se realizó en el mes final de clases, justo antes de los exámenes finales. Una vez finalizada esta etapa se realizó el poblamiento de la base de datos en el programa Excel, para luego ser exportada al software Mplus. 
Tabla 1. Número de alumno, por género, pertenecientes a cada programa de pregrado en ambas universidades participantes

\begin{tabular}{lccccc}
\hline $\begin{array}{l}\text { Institución de Educación } \\
\text { Superior }\end{array}$ & Programa & $\mathrm{N}$ & $\%$ & $\mathrm{~F}$ & $\mathrm{M}$ \\
& Psicología & 70 & 9 & 52 & 18 \\
& Enfermería & 68 & 8.7 & 50 & 18 \\
& Bioingeniería & 28 & 3.6 & 6 & 22 \\
& Educación Parvularia & 30 & 3.8 & 15 & 0 \\
Universidad Tradicional & Medicina Veterinaria & 72 & 9.2 & 44 & 28 \\
& Sociología & 52 & 6.7 & 24 & 28 \\
& Ingeniería Ambiental & 50 & 6.4 & 30 & 20 \\
& Total & 370 & 47.4 & 221 & 134 \\
\hline & Psicología & 54 & 6.9 & 38 & 16 \\
& Enfermería & 136 & 17.4 & 110 & 26 \\
& Ciencias Políticas & 6 & 0.8 & 4 & 2 \\
& Ingeniería Comercial & 38 & 4.9 & 12 & 26 \\
& Arquitectura & 26 & 3.3 & 14 & 12 \\
Universidad Privada & Ingeniería Civil Industrial & 72 & 9.2 & 18 & 54 \\
& Kinesiología & 30 & 3.8 & 18 & 12 \\
& Fonoaudiología & 24 & 3.1 & 12 & 12 \\
& Nutrición y Dietética & 24 & 3.1 & 18 & 6 \\
& Total & 410 & 52.5 & 244 & 166 \\
\hline
\end{tabular}

Tabla 2. Dimensiones e ítems del IPAA

\begin{tabular}{|c|c|}
\hline Dimensiones & Ítems \\
\hline \multirow{5}{*}{ Planificación } & $\begin{array}{l}\text { 1. Hago un plan antes de comenzar a hacer un trabajo escrito. Pienso lo que voy a hacer y lo } \\
\text { que necesito para conseguirlo. }\end{array}$ \\
\hline & 5. Estoy seguro de que soy capaz de comprender lo que me van a enseñar y por eso creo que \\
\hline & voy a tener buenas notas. \\
\hline & 9. Establezco objetivos académicos concretos para cada asignatura. \\
\hline & $\begin{array}{l}\text { 12. Antes de comenzar a estudiar, compruebo si tengo todo lo que necesito: diccionarios, } \\
\text { libros lápices cuadernos fotocopias para no estar siempre interrumpiendo mi estudio }\end{array}$ \\
\hline \multirow{4}{*}{ Ejecución } & 3. Cuando estudio, intento comprender las materias, tomar apuntes, hacer resúmenes, \\
\hline & resolver ejercicios, hacer preguntas sobre los contenidos. \\
\hline & $\begin{array}{l}\text { 6. Cumplo mis horarios de estudio, e introduzco pequeños cambios siempre que es necesario. } \\
\text { 8. Mientras estoy en clase o estudiando, si me distraigo o pierdo el hilo, suelo hacer algo para }\end{array}$ \\
\hline & 10. Busco un sitio tranquilo y donde pueda estar concentrado para estudiar. \\
\hline
\end{tabular}

2. Después de terminar un examen parcial / final, lo reviso mentalmente para saber dónde tuve los aciertos y errores y, hacerme una idea de la nota que voy a tener.

Evaluación

4. Cuando recibo una nota, suelo pensar en cosas concretas que tengo que hacer para mejorar mi rendimiento/ nota media.

7. Guardo y analizo las correcciones de los trabajos escritos o pruebas parciales, para ver dónde me equivoqué y saber qué tengo que cambiar para mejorar.

11. Comparo las notas que saco con los objetivos que me había marcado para esa asignatura. 
Tabla 3. Distribución de participantes por sexo y carrera para el análisis factorial exploratorio y el análisis

\begin{tabular}{|c|c|c|c|c|}
\hline \multirow[t]{2}{*}{ Programa } & $\mathrm{AFE}$ & AFE & $\mathrm{AFC}$ & $\mathrm{AFC}$ \\
\hline & Femenino & Masculino & Femenino & Masculino \\
\hline \multicolumn{5}{|l|}{ Universidad Tradicional } \\
\hline Psicología & 26 & 9 & 26 & 9 \\
\hline Enfermería & 25 & 9 & 25 & 9 \\
\hline Bioingeniería & 3 & 11 & 3 & 11 \\
\hline Educación Parvularia & 15 & 0 & 15 & 0 \\
\hline Medicina Veterinaria & 22 & 14 & 22 & 14 \\
\hline Sociología & 12 & 14 & 12 & 14 \\
\hline Ingeniería Ambiental & 15 & 10 & 15 & 10 \\
\hline \multicolumn{5}{|l|}{ Universidad Privada } \\
\hline Psicología & 19 & 8 & 19 & 8 \\
\hline Enfermería & 55 & 13 & 55 & 13 \\
\hline Ciencias Políticas & 2 & 1 & 2 & 1 \\
\hline Ingeniería Comercial & 6 & 13 & 6 & 13 \\
\hline Arquitectura & 7 & 6 & 7 & 6 \\
\hline Ingeniería & 9 & 27 & 9 & 27 \\
\hline \multicolumn{5}{|l|}{ Industrial } \\
\hline Kinesiología & 9 & 6 & 9 & 6 \\
\hline Fonoaudiología & 12 & 0 & 12 & 0 \\
\hline Nutrición y Dietética & 9 & 3 & 9 & 3 \\
\hline
\end{tabular}

\section{Procedimiento de análisis de datos}

En primer lugar se dividió la muestra, de modo de poder aplicar un análisis factorial exploratorio a una mitad y un análisis factorial confirmatorio a la mitad restante. Esta decisión se tomó, ya que si bien el instrumento ha sido utilizado con población de estudiantes chilenos, los resultados no muestran que la estructura factorial se comporte de la misma manera que la que se creó la escala original en España (Rosário et al, 2007).

Para partir la muestra, y con el objetivo de que ambas partes quedaran equilibradas y no presentaran diferencias importantes, se realizó un análisis de regresión ordinal por ítem de la prueba IPAA, en aquellas variables que podrían relacionarse con la capacidad de autorregulación del aprendizaje (Universidad y carrera de procedencia y sexo). Se evaluó para cada ítem si había diferencias en el nivel de desempeño de los sujetos, en las 3 variables seleccionadas.
El análisis mostró mayores diferencias en el desempeño de los sujetos, en la variable sexo, seguida por la universidad de pertenencia. En general, las mujeres mostraron mayores puntajes de autorregulación. En el caso de las universidades, los resultados fueron más altos en la Universidad privada. En cuanto al programa de pregrado, sólo en 3 ítems se presentaron estas diferencias. Por lo tanto, se decidió utilizar la variable sexo para realizar la partición de la muestra. Para llevar a cabo este procedimiento se construyó una tabla de contingencia, donde se estableció el número de hombres y mujeres que se encuestó en cada uno de los programas. Con los datos de esta tabla se procedió a dividir a los hombres y mujeres de cada carrera, para que quedaran igualmente representados en ambas submuestras. De esta forma también se logró controlar el efecto de las universidades y las carreras, ya que ambas variables quedaron representadas, en ambas mitades, de la misma 
Tabla 4. Estadísticos descriptivos para los ítems de las sub-muestras del análisis factorial confirmatorio y exploratorio

\begin{tabular}{ccccccccccc}
\hline \multirow{4}{*}{ Ítems } & \multicolumn{4}{c}{ Análisis Factorial Exploratorio } & \multicolumn{5}{c}{ Análisis Factorial Confirmatorio } \\
\cline { 2 - 11 } & Media & DS & Simetría & Kurtosis & $\begin{array}{c}\text { Shapiro- } \\
\text { Wilk }\end{array}$ & Media & DS & Simetría & Kurtosis & $\begin{array}{c}\text { Shapiro- } \\
\text { Wilk }\end{array}$ \\
\hline 1 & 3.7 & 1. & -0.4 & -0.4 & $p<.01$ & 3.7 & 1 & -0.5 & -0.3 & $p<.01$ \\
2 & 3.6 & 1.2 & -0.5 & -0.6 & $p<.01$ & 3.6 & 1.2 & -0.5 & -0.6 & $p<.01$ \\
3 & 4 & 0.9 & -0.7 & -0.2 & $p<.01$ & 4.1 & 0.9 & -0.7 & 0 & $p<.01$ \\
4 & 3.8 & 1 & -0.5 & -0.6 & $p<.01$ & 3.7 & 1 & -0.7 & 0.2 & $p<.01$ \\
5 & 3.7 & 0.9 & -0.2 & -0.5 & $p<.01$ & 3.7 & 0.8 & -0 & -0.5 & $p<.01$ \\
6 & 2.7 & 1.1 & 0.4 & -0.4 & $p<.01$ & 2.7 & 1. & 0.3 & -0.4 & $p<.01$ \\
7 & 3 & 1.2 & 0.1 & -0.8 & $p<.01$ & 3.1 & 1.1 & 0 & -0.8 & $p<.01$ \\
8 & 3.4 & 0.9 & -0.1 & -0.3 & $p<.01$ & 3.4 & 1 & -0.3 & -0.4 & $p<.01$ \\
9 & 3.6 & 1.1 & -0.5 & -0.4 & $p<.01$ & 3.5 & 1.1 & -0.5 & -0.4 & $p<.01$ \\
10 & 4.3 & 0.0 & -1.3 & 1.3 & $p<.01$ & 4.3 & 1 & -1.3 & 1.5 & $p<.01$ \\
11 & 3.3 & 1.2 & -0.3 & -0.9 & $p<.01$ & 3.2 & 1.2 & -0.3 & -0.9 & $p<.01$ \\
12 & 3.8 & 1.2 & -0.7 & -0.5 & $p<.01$ & 3.9 & 1.2 & -0.8 & -0.3 & $p<.01$ \\
& & & & & & & & & & \\
\hline
\end{tabular}

forma en que ocurrió en la muestra en general (390 sujetos en cada una). En la Tabla 3, se muestra la distribución de participantes por sexo y carrera para el análisis factorial exploratorio y el análisis factorial confirmatorio.

Los descriptivos para los ítems del IPAA para las sub- muestras del análisis exploratorio y del confirmatorio, se presentan a continuación, en la Tabla 4. Se observa que los distintos estadísticos son muy parecidos entre ambas muestras. Usando el test de Mardia, se evidencia que no existe distribución multivariada normal en la muestra AFE, $\chi_{\text {asimetria }}{ }^{(364)}=790.53, p<.001, Z_{\text {curtosis }}=10.95$, $p<.001$ ni en la AFC, $\chi_{\text {asimetria }}{ }^{(364)}=767.43$, $p<.001, Z_{\text {curtosis }}=9.33, p<.001$

Antes de llevar a cabo los análisis factoriales planificados, se procedió a evaluar si los ítems de la escala IPAA debían ser tratados a nivel intervalar u ordinal. Para esto se analizó la simetría y curtosis, siendo éstas adecuadas para cada uno de los ítems. Luego se aplicó el Test de Normalidad de Shapiro- Wilk, el que mostró que los datos no se comportaban normalmente, por lo tanto los ítems no se pueden tratar como variables numéricas/intervalares. Por esta razón se trabajó a un nivel ordinal, utilizando una matriz de correlaciones policóricas en el Programa M Plus. A continuación se presentan los resultados para cada uno de los análisis realizados.

\section{Resultados}

\section{Análisis Factorial Exploratorio}

Primero, se procedió a evaluar la pertinencia del análisis factorial exploratorio. Para esto se analizó la matriz de correlaciones policóricas sobre la mitad de la muestra seleccionada para este proceso. El estadístico de adecuación muestral de Kaiser-Mayer-Olkin (KMO) fue de 0.87 , lo que indica que la matriz de correlaciones policóricas mostró un buen ajuste a un modelo factorial. Además, Todos los ítems presentaron una Medida de Adecuación de la Muestra (MSA) por sobre .84 , lo que indica a priori que todos serían útiles en la factorización final.

Para decidir el número de factores a retener se utilizaron varios métodos. Todos estos evidenciaron resultados divergentes entre sí. En primer lugar, el criterio Very Simple Structure (VSS), que busca determinar cuál es la mejor solución que considera que cada ítem tributa sólo a 1 o 2 factores, arrojó que la mejor solución para complejidad 1, es de 1 factor, mientras que para complejidad 2, es de 3 factores. El método Minimum Average Partial (MAP) estableció que se alcanza el mínimo con 1 factor. Finalmente, el Método de Paralelo de Horn, sobre 1000 matrices, indicó 4 factores o componentes principales. 
Tabla 5. Matriz de Correlaciones Policóricas para la sub- muestra del análisis factorial exploratorio

\begin{tabular}{|c|c|c|c|c|c|c|c|c|c|c|c|c|}
\hline Ítems & 1 & 2 & 3 & 4 & 5 & 6 & 7 & 8 & 9 & 10 & 11 & 12 \\
\hline 1 & 1 & & & & & & & & & & & \\
\hline 2 & .11 & 1 & & & & & & & & & & \\
\hline 3 & $.36^{* *}$ & $.23^{* *}$ & 1 & & & & & & & & & \\
\hline 4 & $.33^{* *}$ & $.32 * *$ & $.3 * *$ & 1 & & & & & & & & \\
\hline 5 & $.16^{* *}$ & $.13 *$ & $.41 * *$ & $.19^{* *}$ & 1 & & & & & & & \\
\hline 6 & $.34 * *$ & $.21^{* *}$ & $.47 * *$ & $.29^{* *}$ & $.34 * *$ & 1 & & & & & & \\
\hline 7 & $.24 * *$ & $.22 * *$ & $.4 * *$ & $37 * *$ & $.21^{* *}$ & $.4 * *$ & 1 & & & & & \\
\hline 8 & $.29^{* *}$ & $.18^{* * *}$ & $.34 * *$ & $.26^{* * *}$ & $.34 * *$ & $.34^{* *}$ & $.34^{* *}$ & 1 & & & & \\
\hline 9 & $.32 * *$ & $.22 * *$ & $.36^{* *}$ & $.42 * *$ & $.31^{* *}$ & $.48 * *$ & $.39^{* *}$ & $.44 * *$ & 1 & & & \\
\hline 10 & $.33^{* *}$ & .05 & $.4 * *$ & $.23^{* *}$ & $.35^{* * *}$ & $.24^{* *}$ & $.24^{* *}$ & $.32 * *$ & $.33^{* *}$ & 1 & & \\
\hline 11 & $.31^{* *}$ & $.15^{*}$ & $.31 * *$ & $.43^{* *}$ & $.23^{* *}$ & $.39^{* *}$ & $.33^{* *}$ & $.28 * *$ & $.56^{* *}$ & $.24^{* * *}$ & 1 & \\
\hline 12 & $.39^{* *}$ & .1 & $.32 * *$ & $.33^{* *}$ & $.17^{* * *}$ & $.27 * *$ & $.33^{* *}$ & $.2 * *$ & $.28^{* *}$ & $.43^{* *}$ & $.23^{* *}$ & 1 \\
\hline
\end{tabular}

Como método de extracción se utilizó el de Mínimos Cuadrados no Ponderados, debido a que funciona adecuadamente con una matriz de correlaciones policóricas. Como método de rotación se utilizó Promax.

La Tabla 4 muestra las correlaciones de los ítems. La mayoría de las cuales son estadísticamente significativas, positivas y están en el rango de moderadas a débiles, que es lo esperable entre ítems de una escala. Pese a los reiterados intentos de calzar una solución, según los diferentes métodos antes explicados, siempre quedaron cargas cruzadas de distintos factores, dentro de los mismos ítems, lo que refleja que hay una gran relación entre ellos. Esto sugiere la presencia de un factor general que explica la mayor parte de la varianza. Las relaciones que restan, luego de aplicar este factor, son explicadas por factores secundarios o subfactores (Tablas 6).

El procedimiento más adecuado para realizar el análisis factorial de esta escala es una representación bifactorial (Reise, Morizot, \& Hays, 2007). Se trata de un modelo multidimensional de Teoría de Respuesta al Ítem. Es útil para escalas psicométricas que miden constructos amplios y posee una larga historia de aplicación en el ámbito de las habilidades cognitivas.

En este modelo hay un factor general que explica la correlación entre los ítems, además de uno o más factores de grupo, que intentan capturar la covariación de los ítems que es independiente de la correlación explicada en el factor general. Los ítems pueden estar correlacionados porque comparten un rasgo o característica en común, o porque tienen una fuente de covariación conjunta adicional, debido a que comparten contenidos específicos con una parte de los ítems.

En una representación bifactorial se permite que cada ítem de una escala tenga una carga positiva en una dimensión general que subyace a todos los ítems. Típicamente este factor general será más abarcativo a nivel conceptual y a la dimensión que el investigador esté más interesado en medir en los individuos. Adicionalmente, cada ítem puede cargar en cero uno o más factores. Estos factores de agrupamiento serán más estrechos, o específicos teóricamente. En la mayoría de las ocasiones cada ítem carga en sólo uno de estos factores. Ambos tipos de factores, general y específicos, son ortogonales entre sí.

Esto sería lo ocurre en la escala IPAA, aplicada a estudiantes chilenos. La solución ortogonal original explica un $40 \%$ de la varianza de los ítems. El factor general (FG) explica un $58.8 \%$ de la varianza común entre los factores originales, lo que correspondería a un $23.8 \%$ de la varianza total. El subfactor 1 (SF1), explica un $5.9 \%$ de la varianza total, el subfactor 2 (SF2) un $5.3 \%$ y el subfactor 3 (SF3), un 5.4\%. El factor general daría cuenta de la autorregulación del 
Tabla 6. Cargas Factoriales del IPAA en el Análisis Factorial Exploratorio

\begin{tabular}{|c|c|c|c|c|}
\hline Ítem & $\mathrm{FG}$ & SF1 & SF2 & SF3 \\
\hline \multicolumn{5}{|l|}{ Factor 1: Planificación } \\
\hline $\begin{array}{l}\text { 1. Hago un plan antes de comenzar a hacer un trabajo escrito. } \\
\text { Pienso lo que voy a hacer y lo que necesito para conseguirlo. }\end{array}$ & .46 & .32 & .04 & .13 \\
\hline $\begin{array}{l}\text { 10. Busco un sitio tranquilo y donde pueda estar concentrado } \\
\text { para estudiar. }\end{array}$ & .46 & .37 & .28 & -.04 \\
\hline $\begin{array}{l}\text { 12. Antes de comenzar a estudiar, compruebo si tengo todo lo } \\
\text { que necesito: diccionarios, libros, lápices, cuadernos, } \\
\text { fotocopias, para no estar siempre interrumpiendo mi estudio. }\end{array}$ & .46 & .58 & -.0 & .02 \\
\hline
\end{tabular}

Factor 2: Ejecución

3. Cuando estudio, intento comprender las materias, tomar apuntes, hacer resúmenes, resolver ejercicios, hacer preguntas sobre los contenidos.

$\begin{array}{lllllllll}\text { 5. Estoy seguro de que soy capaz de comprender lo que me van } & .41 & -.01 & \mathbf{. 4 8} & & .02\end{array}$ a enseñar y por eso creo que voy a tener buenas notas.

$\begin{array}{llll}.55 & .16 & .38 & .08\end{array}$

8. Mientras estoy en clase o estudiando, si me distraigo o pierdo el hilo, suelo hacer algo para volver a la tarea y alcanzar $\quad .47 \quad \begin{array}{lllll}.4 & .28 & .17\end{array}$ mis objetivos.

6. Cumplo mis horarios de estudio, e introduzco pequeños cambios siempre que es necesario.

$\begin{array}{llll}.55 & .01 \quad .26 & .25\end{array}$

Factor 3: Evaluación

2. Después de terminar un examen parcial / final, lo reviso mentalmente para saber dónde tuve los aciertos y errores y, hacerme una idea de la nota que voy a tener.

4. Cuando recibo una nota, suelo pensar en cosas concretas que tengo que hacer para mejorar mi rendimiento/ nota media.

$\begin{array}{llll}.27 & -.03 & .02 & \mathbf{. 2 0} \\ .50 & .17 & -.12 & . \mathbf{3 5} \\ .49 & .13 & .11 & \mathbf{. 2 3} \\ .62 & -.03 & .11 & \mathbf{. 4 2} \\ .54 & -.02 & -.03 & \mathbf{. 4 4}\end{array}$

7. Guardo y analizo las correcciones de los trabajos escritos o pruebas parciales, para ver dónde me equivoqué y saber qué tengo que cambiar para mejorar

9. Establezco objetivos académicos concretos para cada asignatura.

11. Comparo las notas que saco con los objetivos que me había marcado para esa asignatura.

aprendizaje, como capacidad global del sujeto, mientras que los factores secundarios, agruparían los ítems según la Teoría de Zimmerman (2001), y muy similar a la propuesta de Rosario et al (2007). El subfactor 1 representa el proceso de planificación, el subfactor 2 el proceso de ejecución y el subfactor 3 el proceso de evaluación.

Si bien se evidencian algunas diferencias en las subdimensiones entre la versión original y la solución del modelo bifactorial, las diferencias son coherentes teóricamente. La principal dificultad del análisis se presenta en el ítem 6, que carga tanto en las subdimensiones de Ejecución y de Evaluación. Por motivos teóricos, se decidió que en el Análisis Factorial Confirmatorio debía cargar en la escala de Ejecución, ya que los procesos de evaluación están dirigidos preferentemente a mejorar el desempeño en el proceso de estudio, no en el final de éste.

Los estadísticos descriptivos estos son adecuados, tal como puede observarse en la Tabla 7, ya que si bien no se ajustan a la distribución normal, puesto que todas las pruebas de Shapiro- 
Tabla 7. Estadísticos descriptivos de la Escala IPAA en la solución resultante del AFE

\begin{tabular}{lcccccc}
\hline Factor & Media & DS & Asimetría & Curtosis & Alpha & Shapiro-Wilk \\
\hline General & 3.57 & .59 & -.18 & -.27 & .81 & .06 \\
Planificación & 3.46 & .75 & -.27 & -.46 & .68 & $<.01$ \\
Ejecución & 3.45 & .67 & -.01 & -.32 & .66 & $<.01$ \\
Evaluación & 3.92 & .78 & -.64 & .09 & .59 & $<.01$ \\
\hline
\end{tabular}

Tabla 8. Cargas factoriales del Factor General y Subfactores del IPAA

\begin{tabular}{|c|c|c|c|c|c|}
\hline Factor & Ítems & Estimador & $\mathrm{EE}$ & $\mathrm{Z}$ & Valor- $p$ \\
\hline \multirow{12}{*}{ General } & P1 & .55 & .04 & 12.81 & $<.001$ \\
\hline & $\mathrm{P} 2$ & .39 & .05 & 8.15 & $<.001$ \\
\hline & P3 & .57 & .05 & 12.16 & $<.001$ \\
\hline & $\mathrm{P} 4$ & .43 & .05 & 8.22 & $<.001$ \\
\hline & P5 & .35 & .05 & 6.69 & $<.001$ \\
\hline & P6 & .71 & .03 & 21.07 & $<.001$ \\
\hline & P7 & .48 & .05 & 10.32 & $<.001$ \\
\hline & P8 & .52 & .05 & 11.25 & $<.001$ \\
\hline & P9 & .68 & .04 & 18.83 & $<.001$ \\
\hline & P10 & .51 & .05 & 10.10 & $<.001$ \\
\hline & P11 & .53 & .05 & 11.54 & $<.001$ \\
\hline & $\mathrm{P} 12$ & .47 & .05 & 9.89 & $<.001$ \\
\hline \multirow{3}{*}{ Subfactor 1} & P1 & .13 & .14 & .93 & .35 \\
\hline & P10 & .18 & .18 & .97 & .33 \\
\hline & $\mathrm{P} 12$ & .84 & .84 & 1 & .32 \\
\hline \multirow{4}{*}{ Subfactor 2} & P3 & .80 & 1.27 & .63 & .53 \\
\hline & P5 & -.07 & .12 & -.55 & .58 \\
\hline & P6 & -.09 & .16 & -.54 & .59 \\
\hline & P8 & .11 & .17 & .65 & .51 \\
\hline \multirow{5}{*}{ Subfactor 3} & $\mathrm{P} 2$ & .11 & .06 & 1.75 & .08 \\
\hline & P4 & .34 & .07 & 4.75 & $<.001$ \\
\hline & P7 & -.03 & .06 & -.52 & .60 \\
\hline & P9 & .32 & .06 & 5.01 & $<.001$ \\
\hline & P11 & .72 & .11 & 6.86 & $<.001$ \\
\hline
\end{tabular}

Wilk rechazan la hipótesis nula de normalidad, cuentan con valores de asimetría y curtosis entre 1 y 1 , y los alfa de Cronbach son cercanos a .6 para las escalas específicas y a .8 para la general.

\section{Análisis Factorial Confirmatorio}

Tomando en cuenta el diferente número de factores a retener, arrojado por los indicadores revisados, se decidió probar 2 modelos, uno unidimensional y otro con la estructura bifactorial encontrada en el análisis exploratorio. El objetivo fue definir cuál de los dos modelos se ajustaba mejor a los datos. Para estos fines se utilizaron los datos entregados por la segunda submuestra.

El modelo unidimensional presentó valores de ajuste mediocres $\left(\chi^{2}(54)=162.4 ; p<.001\right.$;
RMSEA=.072; CFI=.931; TLI=.916). Por el contario, el modelo bifactorial mostró un mejor ajuste $\quad\left(\chi^{2} \quad(42)=82.7, \quad p<.01 ; \quad R M S E A=.050\right.$; $C F I=.974 ; T L I=.960)$, siendo tales diferencias estadísticamente significativas ( $\square \chi^{2}(12)=78.143$; $p<.001)$.

En la Tabla 8, se puede observar que el factor general presenta cargas factoriales sobre 0.3 en todos los ítems. En el análisis de los factores secundarios, se puede ver que sólo los ítems 11, 9 y 4 del subfactor 3 (Planificación) son estadísticamente significativos. Las Tabla 8 muestra las cargas factoriales para el Factor General y los Subfactores 1 (Planificación), 2 (Ejecución) y 3 (Evaluación), respectivamente.

En definitiva, todo indica que el instrumento presenta un factor común a todos los ítems, pero existen fuertes dependencias entre grupos de ítems que configuran subfactores menores. A continuación, en la Figura 2, se presenta el modelo bifactorial que resultó del AFC. Si bien las cargas factoriales para los subfactores en Planificación y Ejecución no resultaron significativas, 3 de los 5 en el subfactor de Evaluación si lo fueron. Sin embargo, el hecho que el modelo se ajuste a los factores, nos indica que no se puede descartar que en la población éstos continúen siendo coherentes con las dimensiones teóricas que conforman el constructo de autorregulación del aprendizaje en la Teoría de Zimmerman (2002).

Finalmente, esta solución presenta adecuados estadísticos descriptivos, tal como se presenta en la Tabla 9. Al igual que en el estudio exploratorio, si bien las escalas no provienen de una distribución normal, presentan niveles de asimetría y curtosis entre -1 y 1 , lo que indica distribuciones con forma similar a la normal. Los coeficientes alfa de Cronbach son muy similares a los resultados del estudio exploratorio, con valores cercanos a .6 para las subescalas y a .8 para la escala general. 
Tabla 9. Estadísticos descriptivos de la Escala IPAA en la solución resultante del AFC

\begin{tabular}{lcccccc}
\hline \multicolumn{1}{c}{ Factor } & Media & DS & Asimetría & Curtosis & Alpha & Shapiro-Wilk \\
\hline General & 3.57 & .58 & -.49 & .61 & .79 & $<.01$ \\
Planificación & 3.43 & .74 & -.42 & .15 & .67 & $<.01$ \\
Ejecución & 3.46 & .61 & .14 & -.39 & .57 & $<.01$ \\
Evaluación & 3.95 & .77 & -.91 & .67 & .57 & $<.01$ \\
\hline
\end{tabular}

Es posible afirmar que las subdimensiones son

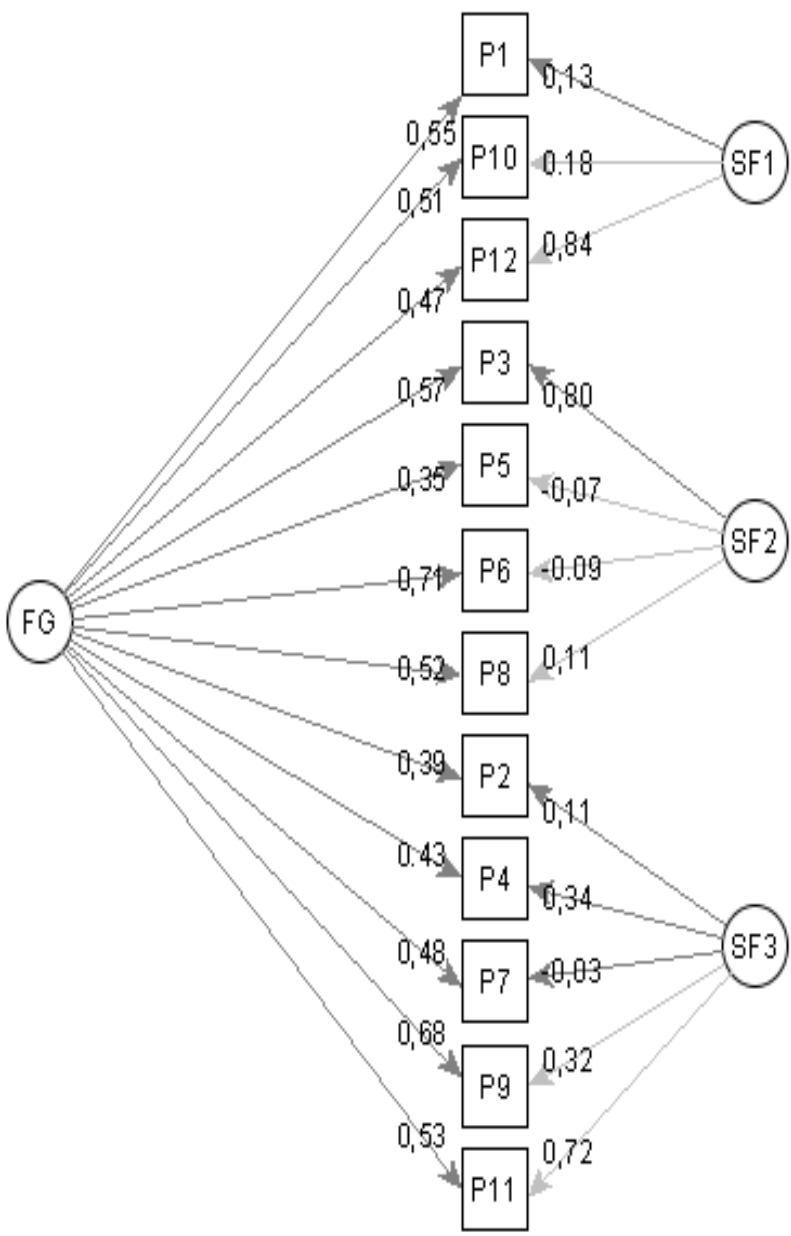

Figura 2. Modelo de la solución bifactorial del IPAA en el Análisis Factorial Confirmatorio

\section{Discusión}

Los resultados de este trabajo, muestran un adecuado funcionamiento del Inventario de Procesos de Autorregulación del Aprendizaje (IPAA), para su aplicación a estudiantes universitarios chilenos. Se evidencia una estructura bifactorial, que da cuenta de la autorregulación como una única capacidad, que a su vez presenta 3 subprocesos cognitivos, tal como es propuesto en el modelo de Zimmerman (2002). coherentes con la Teoría de la Autorregulación de Zimmerman (2002), quien propone que esta capacidad se compone de 3 subprocesos: Planificación, Ejecución y Evaluación. Se concluye que el Subfactor 1, evaluaría las destrezas para planificar el estudio de un aprendiz, mientras que el Subfactor 2 el desempeño mientras se está llevando a cabo, y el Subfactor 3 las conductas que permiten evaluarlo.

En particular, la estructura factorial encontrada hace referencia a una factor general, el cual permitiría medir el constructo de la autorregulación del aprendizaje, como una capacidad general de un individuo. Este factor sería el que explicaría en un mayor porcentaje la varianza. También se muestra la presencia de 3 subfactores, que permitirían evaluar el nivel de cada uno de los elementos que componen teóricamente esta habilidad.

Sin embargo, se observan algunas diferencias con el diseño de la escala española, las que podrían estar relacionadas con una dificultad para trazar límites claros entre los 3 subprocesos que conformar la autorregulación del aprendizaje. Esto es especialmente evidente entre las sub- escalas de Planificación y Ejecución, en las cuales se encuentran las mayores diferencias en las cargas factoriales de ambas versiones de la escala. Lo contrario ocurre con Evaluación, que se mantiene prácticamente igual y es significativa en el análisis factorial confirmatorio.

Los ítems que se comportaron de manera diferente a la escala original fueron el 10, que aquí cargó en la subdimensión de Planificación, mientras que en la versión española formaba parte de Ejecución. Esto podría relacionarse con que buscar un lugar adecuado para el estudio pertenecería a la fase de preparación de Zimmerman (2002), sub etapa de análisis de la tarea, específicamente al proceso de planificación estratégica. También el ítem 5 funcionó diferente, el cual cargó en Ejecución, mientras que en la 
escala original pertenece a Planificación. La seguridad de que se va a aprender podría relacionarse con a la fase de desempeño de Zimmerman (2002), sub etapa de autocontrol, específicamente al proceso de autoinstrucciones. Finalmente el ítem 9, que aquí cargó en la dimensión de Evaluación, en la escala original carga en la dimensión de Planificación. Esto se podría deber a que el proponerse objetivos, como plantea el ítem, podría pertenecer a la fase de Autoreflexión de Zimmerman (2002), sub etapa de autorreacción, específicamente al proceso adaptativo.

De esta manera, el presente estudio se convierte en un aporte en el campo de la medición de la autorregulación del aprendizaje como aptitud, a través del autorreporte, abriendo nuevos espacios para la investigación. Especialmente se propone la validación en otras poblaciones, el diagnóstico de esta capacidad en los universitarios y la valoración del impacto de intervenciones diseñadas para su fomento. Esto es especialmente importante si se considera la significación que ha mostrado este constructo en el campo del aprendizaje.

Una de las limitaciones que muestra esta investigación es que sólo se aplicó a estudiantes provenientes de una región de Chile, por lo que sería relevante replicarlo con muestra que provenga de otros sectores del país.

En relación con lo anterior y teniendo en cuenta la dificultad para establecer límites entre las dos primera subdimensiones, resulta especialmente importante poner a prueba la estructura bifactorial que mostró este estudio con poblaciones diferentes de estudiantes universitarios.

\section{Referencias}

Boekaerts, M., \& Cascallar, E. (2006). How far have we move toward the integration of theory and practice in self-regulation? Educational Psychology Review, 18, 199-210. doi:10.1007/s10648-006-9013-4

Cazan, A. M. (2012). Self-regulated learning strategies-predictors of academic adjustment. Procedia-Social and Behavioral Sciences. 33, 104- 108. doi: 10.1016/jsbspro.2012.01.092.
Cazan, A. M. (2013). Teaching self-regulated learning strategies for psychology students. Procedia-Social and Behavioral Sciences, 78, 743-747.doi: 10.1016/j.sbspro.2013.04.387.

Cassidy, S. (2011). Self-regulated learning in higher education: Identifying key component processes. Studies in Higher Education, 36(8), 989-1000.doi: 10.1080/03075079.2010.503269.

Catañeiras, Guzmán, Posada, Ricchini \& Strucchi, (1999). Sobre estrategias de aprendizaje y hábitos de estudio. Revista Iberoamericana de Diagnóstico y Evaluación - e Avaliação Psicológica, 8(2), 37- 50.

Cerezo, R., Bernardo, A., Esteban, M., Sánchez, M., \& Tuero, E. (2015). Programas para la promoción de la autorregulación en educación superior: Un estudio de la satisfacción diferencial entre metodología presencial y virtual. European Journal of Education and Psychology, 8(1), 30-36.

Cerezo, R., Núñez, J. C., Rosário, P., Valle, A. Rodríguez, S. \& Bernardo, A. B. (2010). New media for the promotion of self-regulated learning in higher education. Psicothema, 22(2), 306-315.

Delors, J. (1996). Learning: The treasure within Report to UNESCO of the International Commission on Education for the Twenty first Century. UNESCO.

Dignath, C., Buettner, G. \& Langfeldt, H-P. (2008). How can primary school students learn self- regulated leraning strategies most effectively? Educational Research Review, 3, 101- 129. doi: 10.1016/j.edurev.2008.02.003.

Dignath- van Ewijk, C. \& Büttner, G. (2008). Components of fostering self-regulated learning among students: A meta-analysis on intervention studies at primary and secondary school level. Metacognition Learning, 3, 231264. doi: 10.1007/s11409-008-9029-x.

Elvira-Valdés, M. A. \& Pujol, L. (2012). Autorregulación y rendimiento académico en la transición secundaria-universidad. Revista Latinoamericana de Ciencias Sociales, Niñez y Juventud, 10(1), 367-378.

European Commission (2001). Making a European área of lifelong learning a reality. Brussels. 
Inan, B., \& Yüksel, D. (2010). Self-regulated learning: How is it applied as a part of teacher training through diary studies? ProcediaSocial and Behavioral Sciences, 3, 116-120. doi:10.1016/j.sbspro.2010.07.021.

Kistner,S., Rakoczy, K., Otto, B., Dignath-van, C., Büttner, G. \& Klieme, E. (2010). Promotion of self-regulated learning in classrooms: Investigating frequency, quality, and consequences for student performance. Metacognition Learning, 5, 157-171. doi: 10.1007/s11409-010-9055-3.

Lanz, M. Z. (2006). Aprendizaje autorregulado: El lugar de la cognición, la metacognición y la motivación. Estudios Pedagógicos, 32(2), 121-132. doi: 10.4067/S0718-070520060002 00007.

Martorell Pallaz, C., \& Gómez Llorenz, O. (2010). Evaluación y enfoque de la Revista de Diagnóstico y Evaluación Psicológica (RIDEP). Revista de Iberoamericana Diagnóstico y Evaluación - e Avaliação Psicológica, 30(2), 35- 56.

Núñez, J. C., Amieiro, N., Álvarez, D., García, T., \& Dobarro, A. (2015). Escala de evaluación de la autorregulación del aprendizaje a partir de textos (ARATEX-R). European Journal of Education and Psychology, 8(1), 9-22.

Núñez, J. C., Cerezo, R., Bernardo, A., Rosário, P, Valle, A., Fernández, E., \& Suárez, N. (2011). Implementation of training programs in selfregulated learning strategies in Moodle format: Results of an experience in higher education. Psicothema, 23(2), 274-281.

Núñez, J. C., Rosário, P., Vallejo, G., \& González-Pienda, J. A. (2013). A longitudinal assessment of the effectiveness of a schoolbased mentoring program in middle school. Contemporary Educational Psychology, 38, 11-21.

Panadero, E., \& Alonso- Tapia, J. (2014). Teorías de autorregulación educativa: una comparación y reflexión teórica. Psicología Educativa, 20, 11-22. doi: 10.1016./j.pse.201 4.05.2002.

Perry, N. E., Hutchinson, L., \& Thauberger, C. (2008). Talking about teaching self-regulated learning: Scaffolding student teachers' development and use of practices that promote self-regulated learning. International Journal of Educational research, 47(2), 97-108. doi: 10.1016/j.ijer.2007.11.010.

Pérez, M. V., Díaz, A., González- Pienda, J. A., \& Núñez, J. C. (2010). Docencia para facilitar el aprendizaje activo y autorregulado. Revista Diálogo Educacional, 10(30), 409-424.

Pintrich, P. (2000). Multiple goals, multiple pathways: The role of goal orientation in selfregulated learning and achievement. Journal of Educational Review, 92, 544-555.

Pintrich, P. (2004). A conceptual framework for assessing motivation and self-regulated learning in college students. Educational Psychology Review, 16(4), 385-407. doi: 1040-726X/04/1200-0385/0.

Pintrich, P., Smith, D., García, T \& Mc Keanie. (1991). A Manual for the Use of the Motivated Strategies for Learning Questionnaire (MSLQ). Michigan: University of Michigan.

Reise, S., Morizot, J. \& Hays, R. (2007). The role of the bifactor model in resolving dimensionality issues in health outcomes measures. Qual Life Res. 16(1), 19- 31.

Rosário, P., Mourao, R., Núñez, J.C., GonzálezPienda, J., Solano, P \& Valle, A. (2007). Eficacia de un programa instruccional para la mejora de procesos y estrategias de aprendizaje en la educación superior. Psicothema. 19, 422- 427.

Rosário, P., Lourenço, A., Paiva, M. O., Núñez, J. C., \& González-Pienda, J. A. (2012). Selfefficacy and perceived utility as necessary conditions for self-regulated academic learning. Anales de psicología, 28, 37-44.

Rosário, P., Núñez, J. C., González-Pienda, J. A., Valle, A., Trigo, L., \& Guimarães, C. (2010). Enhancing self-regulation approaches in firstyear College students: A narrative-based program assessed in the Iberian Peninsula. European Journal of Psychology of Education, 25, 411-428.

Schunk, D. (2005). Self-regulated learning: The educational legacy of Paul R. Pintrich. Educational Psychologist, 40, 85-94.

Smith Castro, V. \& Molina, M. (2011). Cuaderno Metodológico 5. La entrevista cognitiva: guía para su aplicación en la evaluación y mejoramiento de instrumentos de papel y lápiz. San José, CR.: Instituto de 
Investigaciones Psicológicas, Universidad de Costa Rica.

Torrano, F., \& González. M. C. (2004). El Aprendizaje autorrregulado: Presente y futuro de la investigación. Revista Electrónica de Investigación Psicoeducativa, 2(1), 1-34.

Watson, L. (2003). Lifelong Learning in Australia. Canberra: Departament od Education, Science and Training.

Weinstein, C.E. (1987). LASSI User's Manual. Florida: H \& H Publishing.

Winne, P. H. \& Perry, N. E. (2000). Measuring Self- regulated Learning. En Boekaerts, M., Pintrich, P. \& Zeidner (Eds.). Handbook of Self- Regulation. (pp. 531- 566). San Diego: Academic Press.

Zimmerman, B. (1995). Self-regulation involves more than metacognition: A social cognitive perspective. Educational Psychologist, 30(4), 217-221. doi: 10.1207/s15326985ep3004_8.

Zimmerman, B. (2002). Becoming a selfregulated learner: An overview. Theory in to Practice, 41(2), 64-70. 